\title{
Analytical Modeling Enables One to Explain Paradoxical Situations in Behavior and Performance of Electronic Product Materials
}

\section{Suhir $E^{*}$}

Department of Physics, Portland State University, USA

\begin{abstract}
Merits, attributes and challenges associated with the application of analytical (mathematical) predictive modeling in electronics materials science and engineering are addressed, based mostly on the author's research during his tenure with Basic Research, Bell Laboratories and then - with UC-Santa Cruz and Portland State University, Portland, OR, USA. The emphasis is on some practically important, yet paradoxical (i.e., intuitively non-obvious), materials reliability-related situations/phenomena in electronics and optics. It is concluded that all the three basic approaches in Microelectronics and Photonics Materials Science and Engineering - analytical (mathematical) modeling, numerical modeling (simulation) and experimental investigations - are equally important in understanding the physics of the materials behavior and in designing, on this basis, viable and reliable electronic devices and products. As they say, if your only tool is a hammer, all the problems look like nails to you, do they not?
\end{abstract}

\section{Introduction}

\section{Predictive modeling in electronics and photonics material science: role and significance}

Modeling is the major approach of any science, whether pure or applied [1-11]. Research and engineering models can be experimental or theoretical. Experimental models are typically of the same physical nature as the actual phenomenon or the object. They reproduce a notion or an object of interest in a simplified way and often on a different scale. If the latter case similitude criteria are needed to extrapolate the experimental data to the full-scale phenomenon or the object. Some experimental models, analogous models, are based on similarities in the mathematical description of different phenomena or objects. Examples are Prandtl's membrane analogy in the theory of torsion of thin-wall structures; analogy between the motions of a mathematical pendulum and large deflections of flexible rods; some two-dimensional problems in hydrodynamics and theory-of-elasticity.

Theoretical models represent real phenomena using abstract notions. The ultimate goal of a theoretical model is to reveal nonobvious, latent, often even paradoxical, relationships which, although exist, are hidden in the obvious, available and/or experimentally proven input information. The famous relativity theory is a wellknown example of such a model. No theoretical model can provide, of course, results, which are not contained in the input data, in the taken assumptions and in the accepted hypotheses. Experimental models, on the other hand, can lead sometimes to new results. There are examples, when experimentalists discovered phenomena that were different of those they intended to investigate. Perhaps, the most famous one is the surprise discovery of radiation by Antoine Henri Becquerel in 1896 and the birth of nuclear chemistry. A theoretical model can be either analytical or numerical (computational). Analytical models typically employ more or less sophisticated mathematical methods of analysis. The today's numerical models are computer-aided. The most widespread model in the stress-strain evaluations and physical design for reliability of electronic and photonic systems is finite-element analysis (FEA). Experimental and theoretical models have their merits and drawbacks, their areas of application, and should be viewed as equally important and equally indispensable for the design of a viable, reliable, and cost-effective product. One should always try to avoid to be blamed that because his/her only tool is a hammer, all the problems look like nails to him/her. In aerospace, civil, ocean, and in many other areas of engineering, experimental and theoretical models are indeed considered as "equal partners" that complement each other in any significant research or engineering effort. In electronic and photonic engineering the incentive for the development and use of theoretical models is not as great, as it is in the macro-engineering fields. Although during the last two decades the role of theoretical modeling, mostly computer-simulations based, in high-tech engineering has dramatically increased, the situation is still essentially different from the traditional areas of applied science. The overwhelming majority of studies dealing with the physical design and performance of high-tech materials and products are experimental. Electronics and optical physicists and engineers heavily rely, e.g., on thermal cycling tests to check and assure robustness of their designs and products. Some leading electronics materials and reliability journals are reluctant to publish theoretical/modeling papers, or at least contributions not supported by an experiment [11]. There are several objective reasons for that. First, experiments could be carried out with "full autonomy", i.e. without necessarily requiring theoretical support. Unlike theory, testing can be, and, in effect, is used as a final proof of the viability and reliability of a product. Testing procedures are essential requirements of military and commercial specifications for such products. Second, experiments in the "high-tech" field, expensive as they are, are considerably less costly than, e.g., those in naval architecture, or in aerospace field, or in civil engineering, where "specimens" might cost millions of dollars. Third, "high-tech" experimentations are much easier to design, organize, and conduct than in the macro-engineering world. Fourth, in "high-tech" engineering materials, whose properties are not completely known, are often and successfully employed in products. Lack of information about

*Corresponding author: Suhir E, Professor, Department of Physics, Portland State University, USA, Tel: 503-725-3806; E-mail: suhire@aol.com

Received November 05, 2015; Accepted February 29, 2016; Published March 03, 2016

Citation: Suhir E (2016) Analytical Modeling Enables One to Explain Paradoxical Situations in Behavior and Performance of Electronic Product Materials. J Phys Math 7: 161. doi:10.4172/2090-0902.1000161

Copyright: (c) 2016 Suhir E. This is an open-access article distributed under the terms of the Creative Commons Attribution License, which permits unrestricted use, distribution, and reproduction in any medium, provided the original author and source are credited. 
the material properties is often viewed as an obstacle for implementing theoretical modeling, including FEA. The last, but not the least reason is that many of the leading specialists in "high-tech" engineering (experimental physicists, materials scientists, chemists, chemical engineers) traditionally use experimental methods as their major research tool. Many of them simply do not feel that adding theoretical modeling will make an appreciable difference in the state-of-the-art of what they do. It is not just a coincidence that eleven out of twelve Bell Labs Nobel laureates were experimentalists.

On the other hand, the application of experimental investigations, unlike theoretical modeling, requires, as a rule, considerable time and is often associated with significant expense. What is even more important though is that experimental data inevitably reflect the effect of the combined action of a variety of factors affecting the phenomenon or the product of interest, and is usually insufficient to understand the behavior and performance of a material or a device? Such a lack of insight often leads to tedious, time-consuming and costly experimental efforts. Experimental data typically cannot be simply extended to new situations or designs that are different from those already tested. It is always easy to recognize purely empirical relationships obtained by formal processing of experimental data and not based on any rational (theoretical) considerations, underlying the physical nature of the phenomenon of interest. These relationships contain fractional exponents and coefficients, odd units, etc. Although such relationships may have a certain practical value, the very fact of their existence should be attributed to the lack of knowledge in the given area of applied science. Typical examples are a power law (e.g., the one used in prooftesting of optical fibers, when their delayed fracture, aka "static fatigue", is evaluated) or an inverse power law (e.g., numerous relationships of Coffin-Manson type, when temperature cycling is used to evaluate the lifetime of solder joint interconnections). In view of the above, it has to be admitted that a good theoretical model could be as practical and as a meaningful as a thoroughly conducted experimentation.

Here is what could be gained by using theoretical modeling as an additional effective resource in electronics and photonics materials science and engineering:

1) Unlike experimentation, predictive modeling is able to shed light on the role of each particular parameter that affects the behavior and performance of the object, material, or a product of interest;

2) Although testing can reveal insufficiently robust elements, it is incapable to detect superfluously reliable ones; "over-engineered" (superfluously robust) objects may have excessive weight and be more costly than necessary; in mass production of expensive high-quality products, superfluous reliability may entail substantial and unnecessary additional costs;

3) Theoretical modeling can usually predict the result of an experiment in less time and at smaller expense than it would take to perform the actual experiment;

4) In many cases, theory serves to discourage wasting time on useless experiments; numerous attempts to build impossible heat engines have been prevented by a study of the theoretical laws of thermodynamics; while this is, of course, a classical and an outstanding example of the triumph of a theory, there are also numerous, though less famous, examples, when plenty of time and expense were saved because of prior theoretical modeling of a problem;

5) In the majority of research and engineering projects, a preliminary theoretical analysis enables one to obtain valuable information about the phenomenon or the object to be investigated, and gives an experimentalist an opportunity to decide, what and how should be tested or measured, and in what direction success might be expected;

6) By shedding light on "what affects what", theoretical/predictive modeling often serves to suggest new experiments. E.g., the theoretical analyses of stresses in bi-material assemblies (see Ref.1 in section 1) and in semiconductor thin films (see Ref. 1 in section 8) triggered numerous experimental investigations aimed at the rational physical design of semiconductor crystal grown systems;

7) Theory can be used to interpret empirical results and to bridge the gap between different experiments and to extend the existing experience on new materials, components and structures.

8) One cannot do without a good theory when developing rational (optimal) designs. The idea of optimization of structures, materials, functions and costs has penetrated many areas of modern engineering. No progress in this direction could be achieved without application of theoretical methods.

\section{References}

1. Suhir E (1997) The Future of Microelectronics and Photonics and the Role of Mechanics and Materials. Proceedings of the Electronic Packaging and Technology Conf EPTC'97, Singapore.

2. Suhir E (1999) Thermal Stress Failures in Microelectronics and Photonics: Prediction and Prevention. Future Circuits International.

3. Suhir E (2000) Microelectronics and Photonics - the Future. Microelectronics Journal 31: 839-581.

4. Suhir E (2001) Thermo-Mechanical Stress Modeling in Microelectronics and Photonics. Electronics Cooling 7: 01-28

5. Suhir E, Wong CP, Lee YC (2008) Micro- and Opto-Electronic Materials and Structures: Physics, Mechanics, Design, Packaging, Reliability. Springer.

6. Suhir ED, Steinberg T, Yi (2011) Dynamic Response of Electronic and Photonic Systems to Shocks and Vibrations, John Wiley.

7. Suhir E (2011) Linear Response to Shocks and Vibrations. In: Suhir ED Steinberg, Yu T (eds.) Structural Dynamics of Electronic and Photonic Systems, John Wiley, Hoboken, NJ, USA.

8. Suhir E (2011) Thermal Stress Failures: Predictive Modeling Explains the Reliability Physics Behind Them, IMAPS Advanced Microelectronics, vol. 38, No.4.

9. Suhir E (2011) Predictive Modeling of the Dynamic Response of Electronic Systems to Shocks and Vibrations, ASME Appl. Mech. Reviews, vol. 63, No. 5

10. Suhir E (2011) Predictive Modeling is a Powerful Means to Prevent Thermal Stress Failures in Electronics and Photonics, ChipScale Reviews, vol.15, No. 4.

11. Suhir E (2012) Thermal Stress in Electronics and Photonics: Prediction and Prevention, Keynote presentation, Therminic, Budapest.

Citation: Suhir E (2016) Analytical Modeling Enables One to Explain Paradoxical Situations in Behavior and Performance of Electronic Product Materials. J Phys Math 7: 161. doi:10.4172/2090-0902.1000161 